\title{
MR imaging and targeting of a specific alveolar macrophage subpopulation in LPS-induced COPD animal model using antibody-conjugated magnetic nanoparticles
}

\author{
This article was published in the following Dove Press journal: \\ International Journal of Nanomedicine \\ 24 March 2014 \\ Number of times this article has been viewed
}

\author{
Achraf Al Faraj' \\ Asma Sultana Shaik' \\ Sibtain Afzal ${ }^{2}$ \\ Baraa Al Sayed' \\ Rabih Halwani² \\ 'King Saud University, College of \\ Applied Medical Sciences, Department \\ of Radiological Sciences, Molecular \\ and Cellular Imaging Lab, Riyadh, \\ Saudi Arabia; ${ }^{2}$ King Saud University, \\ Prince Naif Center for Immunology \\ Research, Asthma Research Chair, \\ College of Medicine, Riyadh, Saudi \\ Arabia
}

\begin{abstract}
Purpose: Targeting and noninvasive imaging of a specific alveolar macrophage subpopulation in the lung has revealed the importance for early and better diagnosis and therapy of chronic obstructive pulmonary disease (COPD). In this study, the in vivo effect of pulmonary administration of iron oxide nanoparticles on the polarization profile of macrophages was assessed, and a noninvasive free-breathing magnetic resonance imaging (MRI) protocol coupled with the use of biocompatible antibody-conjugated superparamagnetic iron oxide (SPIO) nanoparticles was developed to enable specific targeting and imaging of a particular macrophage subpopulation in lipopolysaccharide-induced COPD mice model.
\end{abstract}

Materials and methods: Enzyme-linked immunosorbent assay, Real-time polymerase chain reaction, and flow cytometry analysis were performed to assess the biocompatibility of PEGylated dextran-coated SPIO nanoparticles. Specific biomarkers for M1 and M2 macrophages subsets were selected for conjugation with magnetic nanoparticles. MRI protocol using ultra-short echo time sequence was optimized to enable simultaneous detection of inflammation progress in the lung and detection of macrophages subsets. Flow cytometry and immunohistochemistry analysis were finally performed to confirm MRI readouts and to characterize the polarization profile of targeted macrophages.

Results: The tested SPIO nanoparticles, under the current experimental conditions, were found to be biocompatible for lung administration in preclinical settings. Cluster of differentiation (CD)86- and CD206-conjugated magnetic nanoparticles enabled successful noninvasive detection of M1 and M2 macrophage subpopulations, respectively, and were found to co-localize with inflammatory regions induced by lipopolysaccharide challenge. No variation in the polarization profile of targeted macrophages was observed, even though a continuum switch in their polarization might occur. However, further confirmatory studies are required to conclusively establish this observation.

Conclusion: Coupling of magnetic iron oxide nanoparticles with a specific antibody targeted to a particular macrophage subpopulation could offer a promising strategy for an early and better diagnosis of pulmonary inflammatory diseases using noninvasive MRI.

Keywords: magnetic resonance imaging, MRI, lung imaging, lung inflammation, iron oxide nanoparticles, macrophage tracking, lipopolysaccharide

\section{Introduction}

Predicted to be the third leading cause of death, and the fifth leading cause of disability by 2020, chronic obstructive pulmonary disease (COPD) will certainly impose a greater burden on the health care system globally. ${ }^{1}$ Therefore, new methodologies 
are required to better assess the pathological and physiological impairments of this progressive disease and to provide alternative diagnosis and therapies.

Studies in experimental animals and clinical settings have led to the concept that alveolar macrophages (AMs) play a central role in the pathogenesis of COPD as a major source of mediators. ${ }^{2,3}$ AMs are among the most abundant cells in the respiratory tract and are characterized by considerable diversity and plasticity in response to various signals within this environment. AMs undergo classical pro-inflammatory M1 activation, which is characterized by the expression of high levels of pro-inflammatory cytokines, high production of reactive nitrogen and oxygen intermediates, promotion of T helper-1 response, and strong tumoricidal and microbicidal activity. Alternatively, they may have an immunoregulatory M2 activation, which might be implicated in parasite containment and promotion of tissue remodeling and disease progression., ${ }^{4,5}$

Although progress has been made to characterize the surface phenotype, activating signals and molecular pathways associated with different forms of macrophage activation, full delineation of this diversity under in vivo conditions requires further investigation. Therefore, imaging and targeting of a specific AM subpopulation in COPD will be of great importance to understand the molecular events involved in its pathophysiology and/or help in early and better diagnosis.

Among the different strategies for inflammation imaging, molecularly targeted nanoparticles have been recognized as the most promising to achieve breakthroughs in health care. ${ }^{6,7}$ Magnetic nanoparticles possess much versatility that make them well suited for these applications by having the potential to enable early detection and prevention, and essentially improve diagnosis, treatment, and follow-up of diseases. ${ }^{8}$ They are widely utilized in translational cell- and animal-based experiments, and some of these nanomaterials are now US Food and Drug Administration (FDA) approved for human use. ${ }^{9,10}$

Macrophage imaging using magnetic resonance imaging (MRI), coupled with the use of iron oxide magnetic nanoparticles, has emerged in recent times as a promising noninvasive technique for preclinical and clinical studies of several inflammatory diseases. ${ }^{11}$ This technique has been successfully used in different preclinical and clinical applications such as atherosclerosis and myocardial infarction, stroke, multiple sclerosis, rheumatoid arthritis, and kidney transplantation. ${ }^{12}$ However, limited investigations of inflammation and macrophage trafficking in the lung using imaging technologies has been performed because of the difficulties in imaging this organ (ie, signal loss due to cardiac pulsation and respiration, susceptibility artifacts caused by multiple air-tissue interfaces, and low proton density). With the ongoing technical improvements in gradient systems and development of MRI pulse sequence techniques, ${ }^{13,14}$ noninvasive detection using MRI may open new perspectives for imaging, diagnosis, and treatment of respiratory diseases such as COPD.

We have recently reported the possibility of noninvasive tracking of macrophage subsets in an inflammatory model using high resolution MRI after their intravenous administration. ${ }^{15}$ In addition, we have shown in another study that the ex vivo labeling of macrophage subpopulations with iron oxide nanoparticles did not affect their polarization and proliferation. ${ }^{16}$ It was proposed that coupling of iron oxide nanoparticles with a specific antibody targeted to a particular subpopulation of macrophages could offer a promising strategy for an early and better diagnosis of different inflammatory diseases using noninvasive MRI. Therefore, the purpose of the current study was to assess the in vivo effect of intrapulmonary administration of superparamagnetic iron oxide (SPIO) nanoparticles on AM polarization profiles in a COPD model and to develop a noninvasive MRI protocol to specifically target and monitor a macrophage subpopulation using specific antibody-conjugated SPIO nanoparticles.

\section{Material and methods Animals and COPD model}

Female BALB/c mice (20-22 g) were obtained from the University's main animal care center. All experiments were performed in accordance with the National guidelines for the care of laboratory animals, and the study was approved by the Ethical Committee of the College of Applied Medical Sciences (agreement number: CAMS05/3334). COPD model was induced by intrapulmonary instillation $\left(0.5 \mathrm{mg} \cdot \mathrm{kg}^{-1}\right.$; volume $[\mathrm{V}]=100 \mu \mathrm{L}$ ) of lipopolysaccharide (LPS) from Escherichia coli (Santa Cruz Biotechnology, Inc., Santa Cruz, CA, USA) using a MicroSprayer ${ }^{\circledR}$ aerosolizer (PennCentury Inc., Glenside, PA, USA).

\section{SPIO nanoparticles and lung exposure}

SPIO nanoparticles used in this study were coated with $40,000 \mathrm{~g} / \mathrm{mol}$ dextran and functionalized by the addition of $300 \mathrm{~g} / \mathrm{mol}$ polyethylene glycol (PEG) chain (Micromod Partikeltechnologie GmbH, Rostock, Germany). They were previously characterized, and their in vitro biocompatibility to macrophage subsets was extensively assessed. ${ }^{16}$

To assess the in vivo biological impact of intrapulmonary administration of SPIO nanoparticles on the polarization 
profile of AMs in LPS-induced COPD lung, animals were divided into four groups ( $\mathrm{n}=6$ for each group): $\mathrm{G}_{\mathrm{ctrl}}$, control mice intrapulmonary instilled with physiological saline solution ( $\mathrm{V}=100 \mathrm{~mL}$ ); $\mathrm{G}_{\mathrm{LPS}}$, lung inflammation-bearing mice intrapulmonary instilled with LPS as a COPD model; $\mathrm{G}_{\text {SPIO }}$, control mice intrapulmonary instilled with iron oxide nanoparticles $([\mathrm{Fe}]=4 \mathrm{mM}$, corresponding to $16 \mathrm{mmol}$ of iron per kilogram; V $=100 \mu \mathrm{L}$ ) using the MicroSprayer ${ }^{\circledR}$ aerosolizer 24 hours post-LPS challenge; $\mathrm{G}_{\text {LPS-SPIO }}$, mice instilled with LPS and with SPIO 24 hours post-LPS challenge.

\section{Isolation of pulmonary macrophages}

Mice were sacrificed by overdose of intraperitoneal pentobarbital injection, their tracheas cannulated, and lungs lavaged three times with $1 \mathrm{~mL}$ phosphate-buffered saline (PBS) containing $0.6 \mathrm{mM} / \mathrm{L}$ ethylenediaminetetraacetic acid (EDTA) at 24 hours post-SPIO instillation corresponding to 48 hours post-LPS challenge. The supernatant was directly stored at $-80^{\circ} \mathrm{C}$ for cytokines analysis. Inflammatory cell infiltration was determined by pooling lavaged samples from each mouse and counting cells using a Scepter ${ }^{\mathrm{TM}}$ automated cell counter (Merck Millipore, Billerica, MA, USA).

Twenty thousand bronchoalveolar lavage (BAL) cells/ samples were cytospun and affixed to glass slides in $-20^{\circ} \mathrm{C}$ methanol for 3 minutes. Cells were stained with WrightGiemsa and differentially classified on the basis of nuclear morphology as neutrophils, macrophages, or lymphocytes. As the volume of BAL fluid (BALF) lavaged from each mouse varies between animals and groups, BAL data were expressed as cells/mL BALF.

\section{Assessment of cytokine levels}

Analyses of interleukins (ILs) and chemokines were performed on the BALF supernatants obtained from the different treatment groups. The levels of IL-12 and CXCL-10 (chemokine [C-X-C motif] ligand 10) as markers of M1-polarized macrophages and the levels of IL-4 and CCL-22 (chemokine [C-C motif] ligand 22) as markers of M2-polarized macrophage ${ }^{17}$ were quantified by an enzyme-linked immunosorbent assay (ELISA) as per the manufacturer's protocol (R\&D Systems, Abingdon, UK).

\section{Real-time TaqMan ${ }^{\circledR}$ polymerase chain reaction (PCR) analysis}

To evaluate the relative gene expression profiles, BALF cell pellets were first incubated with TRIzol ${ }^{\circledR}$ LS reagent
(Life Technologies, Carlsbad, CA, USA), according to the manufacturer's specifications and then stored at $-80^{\circ} \mathrm{C}$. RNA (ribonucleic acid) was then reverse-transcribed into complementary deoxyribonucleic acid (cDNA) using M-MLV reverse transcriptase (Promega Corporation, Maddison, WI, USA). The cDNA was then amplified by real-time quantitative TaqMan ${ }^{\circledR}$ PCR using an ABI Prism 7700 (both Applied Biosystems, CA, USA) sequence detection system. Glyceraldehyde 3-phosphate dehydrogenase was analyzed as an internal control. SYBR Green Master PCR mix (Applied Biosystems) was used to amplify nitric oxide synthase (NOS)-2, CXCL-10, and tumor necrosis factor (TNF) as markers of M1 macrophages, and CCL-17, CCL-22, arginase 1 (Arg1), and IL-10 primers (Integrated DNA Technologies, Leuven, Belgium) as markers of M2 macrophages. Primers for NOS-2 were 5'-CGCAGCTGGGCTGTACCAA-3' and 5'-TGATGTTTGCTTCGGACATCA-3'; for CXCL-10, 5'-GCT GCCG TCAT T T TC T GC-3' and 5'-TCTCACTGGCCCGTCATC-3'; for T NF, $\quad 5^{\prime}$ - C T G TA G C C C A C G T C G TA G C - 3' and 5'-TTGAGATCCATGCCGTTG-3'; for CCL-17, 5' - T GC T T C T GGGGACT T T T C T G-3' and 5'-GAATGGCCCCTTTGAAGTAA-3'; for CCL-22, 5' - TCT TGCTGTGGCAATTCAGA-3' and 5'-GAGGGTGACGGATGTAGTCC-3'; for Arg 1, 5'-GAATCTGCATGGGCAACC-3' and 5'-GAATCCTGGTACATCTGGGAAC-3'; and for IL-10, 5'-GAAGCATGGCCCAGAAATCA-3' and 5'-TGCTCCACTGCCTTGCTCTT-3'. The gene expressions in SPIO, LPS, and LPS-SPIO mice were presented as a fold increase in transcript expression compared with control mice. The fold difference in mRNA (messenger RNA) expression between the different groups was determined using a software program developed by Applied Biosystems.

\section{Flow cytometry analyses of BALF}

BALF from another set of animals ( $n=3$ for each) was seeded in cell culture flask to enable adherence of macrophages for 2 hours. Macrophages were then scraped, and flow cytometry analysis was performed to characterize the AM subpopulations based on their surface membrane receptors. Cells were stained with the following anti-mouse antibodies: fluorescein isothiocyanate (FITC)-labeled anti-cluster of differentiation $11 \mathrm{~b}$ (CD11b), Alexa Fluor ${ }^{\circledR}$ (Life Technologies) 488-labeled anti-CD86, Alexa Fluor ${ }^{\circledR}$ 647-labeled anti-CD197, FITClabeled anti-CD206 and RPE (R-phycoerythrin)-labeled anti-CD150 (AbD Serotec, Kidlington, UK) and analyzed 
with BD ${ }^{\text {TM }}$ LSR II flow cytometer using DIVA (BD Biosciences, San Jose, CA, USA) software.

\section{Antibody-conjugated SPIO functionalization}

To specifically target one subpopulation of macrophages in the lungs of COPD mice, and enable their noninvasive tracking using noninvasive MRI, specific M1 and M2 antibodyconjugated SPIO nanoparticles were developed.

Thiolated CD86 or CD206 antibodies (R\&D Systems) targeted to M1 and M2 macrophage subsets, respectively, were conjugated to maleimide-functionalized SPIO nanoparticles. Sulfo-SMCC (4-(N-Maleimidomethyl)cyclohexane-1carboxylic acid 3-sulfo-N-hydroxysuccinimide ester) cross-linker was first added to SPIO nanoparticles with CLD$\mathrm{NH}_{2}$ (aminated cross-linked dextran) surface to introduce maleimide groups as previously described. ${ }^{18}$ Briefly, sulfoSMCC solution (100 $\mu \mathrm{L}$ of $14.35 \mu \mathrm{M})$ in DMSO (dimethyl sulfoxide) was added to $5 \mathrm{mg}$ of iron oxide nanoparticles in PBS-EDTA buffer under gentle shaking for 1 hour at room temperature. After incubation, the suspension was washed with PBS-EDTA buffer with PD (protein desalting)-10 sizeexclusion columns to remove unreacted sulfo-SMCC. Then, the antibody conjugation to iron oxide nanoparticles was accomplished by the addition of maleimide-functionalized particles to the sulfhydryl-labeled antibody and incubation under gentle shaking for 3 hours at room temperature. The functionalization was performed under optimized conditions (ie, $\mathrm{pH}$ and dilution) to ensure homogeneous functionalization. Remaining functional groups were blocked by the addition of $100 \mu \mathrm{L}$ of $20 \mathrm{mM}$ freshly prepared cysteine solution. Finally, the antibody-conjugated SPIO nanoparticles were purified on magnetic columns (MACS Separator; Miltenyi Biotec, Bergisch Gladbach, Germany).

\section{Quantification of conjugated antibodies and measurement of nanoparticle size}

The amount of CD86 or CD206 antibodies conjugated to SPIO nanoparticles was measured using a modified bicinchoninic acid (BCA) protein assay that involves the reduction of $\mathrm{Cu}^{2+}$ to $\mathrm{Cu}^{+}$by proteins and the formation of a purple-blue copper-protein complex in alkaline environment as previously described. ${ }^{19}$ Briefly, the calibration curve was obtained by adding increasing amounts of the CD86 or CD206 antibodies (1.6-25.0 $\mu \mathrm{g} / \mathrm{mL})$ to aminated iron oxide nanoparticles at a constant iron concentration of $0.5 \mathrm{mg} / \mathrm{mL}$. The antibodyconjugated nanoparticles were adjusted to the same iron concentration of $0.5 \mathrm{mg} / \mathrm{mL}$ and developed with BCA reagent
(Thermo Fisher Scientific, Bremen, Germany) together with the calibration curve for 2 hours at $37^{\circ} \mathrm{C}$. Absorbance of the reaction products was measured at $\lambda=562 \mathrm{~nm}$ using a Sunrise $^{\mathrm{TM}}$ (Tecan, Crailsheim, Germany) microplate reader.

After filtration of the probe through a $0.22 \mu \mathrm{m}$ polyethersulfone syringe filter, the hydrodynamic size of the nanoparticles was measured before and after antibody conjugation using a Zetasizer Nano ZS90 (Malvern Instruments, Malvern, UK) at constant iron concentration of $0.2 \mathrm{mg} / \mathrm{mL}$ in PBS buffer.

\section{MRI relaxivity measurements}

The relaxivities $(\mathrm{r} 1, \mathrm{r} 2$, and $\mathrm{r} 2 *)$ of the different nanoparticles were measured at $25^{\circ} \mathrm{C}$ on tubes containing suspension of nanoparticles at different iron concentrations $(0,0.10,0.15$, 0.20, 0.40, 0.60, and 0.80 mM) using a 4.7T PharmaScan 47/16 Bruker magnet interfaced to ParaVision 5.1 software (Bruker Biospin GmbH, Rheinstetten, Germany). For the measurement of T1 relaxation time, an IR-FISP (inversion-recovery fast imaging with steady state precession) sequence was used with repetition time $(\mathrm{TR}) / \mathrm{echo}$ time $(\mathrm{TE})=4 / 2 \mathrm{~ms}$, and an increasing TI (inversion time) starting from TI $=100 \mathrm{~ms}$ with 60 echoes. For the measurement of T2 relaxation time, an MSME (multispin multi-echo) sequence was used with a TR of 2,500 ms and increasing TEs starting from $12 \mathrm{~ms}$, with 15 echoes of 12 $\mathrm{ms}$ echo spacing. For the measurement of $\mathrm{T} 2 *$ relaxation time, an MGE (multi-gradient echo) sequence was used with a TR of 1,500 ms and increasing TEs starting from $4 \mathrm{~ms}$, with 15 echoes of $4 \mathrm{~ms}$ echo spacing. T1, T2, and T2* relaxation times were automatically calculated using the ParaVision ${ }^{\circledR}$ (Bruker Biospin $\mathrm{GmbH}$ ) image analysis software. Relaxivities were then calculated as the slope of the linear regression generated from a plot of the measured relaxation rate $\left(1 / \mathrm{Ti}\right.$, where $\mathrm{i}=1,2$, or $\left.2^{*}\right)$ versus the concentration of the particles, ie, $(1 / \mathrm{Ti})=(1 / \mathrm{Ti}(0))+$ ri[SPIO], where Ti denotes the relaxation times of a suspension containing the particles, and $\operatorname{Ti}(0)$ is the relaxation time of the solvent (water) without particles.

\section{Noninvasive detection using MRI}

To noninvasively detect M1 or M2 macrophage subpopulations targeted with either CD86- or CD206-conjugated SPIO nanoparticles and monitor their biodistribution in LPS-induced COPD model, a free-breathing imaging protocol using a radial ultra-short TE (UTE) sequence $(\mathrm{TR} / \mathrm{TE}=100 / 0.4 \mathrm{~ms}$, four averages, total acquisition time $=5 \mathrm{~min} 21 \mathrm{~s}$ ) with $100 \mu \mathrm{m} \times 100 \mu \mathrm{m}$ pixel resolution and $1 \mathrm{~mm}$ axial slice thickness was used. This protocol enabled simultaneous detection of antibody-conjugated magnetic nanoparticles targeted to 
macrophages and the visualization of inflammation progression in the lung. Pulmonary MRI protocol was performed on mice $(n=10)$ before LPS induction (control group), 48 hours post-LPS challenge, and 2 hours post-intrapulmonary administration of either non-conjugated (LPS + SPIO), CD86conjugated (LPS + CD86-SPIO), or CD206-conjugated (LPS + CD206-SPIO) SPIO nanoparticles. Based on preliminary studies (data not shown), 2 hours post-intrapulmonary administration of nanoparticles was found sufficient to enable specific targeting and therefore was chosen for MRI and investigation of the polarization profile of AMs.

Image analysis was performed as previously described. ${ }^{20}$ Briefly, the regions of interest were drawn around apparent vascular structures, and these regions were subtracted from the map to retain lung parenchyma. Threshold was chosen to include all apparent fluid signals in lung parenchyma. The number of hyperintensity pixels was averaged on a set of ten slices, with $0.5 \mathrm{~mm}$ inter-slices positioned at the same position in lung parenchyma for all animals.

\section{Characterization of targeted macrophages using flow cytometry}

Macrophages were obtained from mouse lungs $(n=5)$ after MRI protocol at 2 hours post-antibody-conjugated SPIO nanoparticle administration. They were prepared for flow cytometry as per the protocol described elsewhere. ${ }^{21}$ Briefly, the dissected lungs were held in complete RPMI (Roswell Park Memorial Institute) medium supplemented with $25 \mathrm{mM}$ HEPES (2-[4-(2-hydroxyethyl)piperazin-1-yl] ethanesulfonic acid). Lungs were minced in complete medium, and the filtrates were centrifuged (1,500 rpm for 5 minutes). The number of cells in the supernatant was determined, and then the solutions were passed through magnetic activated cell sorter (MACS) microbeads and through MACS LD or MS columns, as per the manufacturer's protocol (Miltenyi Biotec). The eluted unbound and bound cell fractions were first separated, and after a repeat cell count, they were tested for different markers by flow cytometry. The cells were then washed, fixed, and permeabilized with Cytofix/Cytoperm ${ }^{\mathrm{TM}}$ (BD Biosciences). They were stained with either Alexa Fluor ${ }^{\circledR}$ 488-labeled antiCD86 or FITC-labeled anti-CD206 anti-mouse antibodies and analyzed with the BD ${ }^{\mathrm{TM}}$ LSR II flow cytometer.

\section{Lung immunohistochemistry and Prussian blue staining}

Lungs $(n=5)$ were removed and fixed overnight in $4 \%$ paraformaldehyde. They were then embedded in paraffin for histological analysis, and sets of four consecutive $5 \mu \mathrm{m}$ thick sections were obtained. Immunohistochemistry was then performed using the following primary antibodies: F4/80 rat monoclonal immunoglobulin G (1:100), NOS-2 rabbit polyclonal antibody $(1: 1,000)$, and arginase goat polyclonal antibody (1:100) (Santa Cruz Biotechnology, Inc.). Respective mouse avidin biotin complex (ABC) staining systems were used as secondary antibodies according to the manufacturer's protocol. Briefly, tissue sections were deparaffinized and incubated with primary antibodies diluted in blocking sera. Slides were then washed and incubated with biotinylated secondary antibodies followed by enzyme reaction with addition of peroxidase substrate. The sections were counterstained with hematoxylin, dehydrated in a series of alcohol and xylene solutions, mounted, and observed under a BX53 (Olympus Corporation, Tokyo, Japan) microscope.

To check for the presence of antibody-conjugated SPIO nanoparticles and their co-localization with macrophages, a Prussian blue staining protocol was followed. Sections were stained in a 1:1 solution of $20 \%$ aqueous hydrochloric acid and $10 \%$ aqueous solution of potassium ferrocyanide. They were then counterstained with neutral red, dehydrated with a series of alcohols, cleared in xylene, and observed after mounting.

\section{Statistical analysis}

Data presented as the mean standard deviation were analyzed by $t$-test using SPSS v 12.0 (SPSS Inc., Chicago, IL, USA) software. A $P$-value $<0.05$ was considered significant for all tests.

\section{Results Effect of SPIO and/or LPS intrapulmonary exposure on the polarization state of AMs}

Intrapulmonary instillation of LPS $\left(0.5 \mathrm{mg} \cdot \mathrm{kg}^{-1} ; \mathrm{V}=100 \mu \mathrm{L}\right)$ was found to induce significant pulmonary inflammatory responses related to alterations in lung capacity and mechanics as observed in COPD. Accordingly, the peak influx of total inflammatory cells was detected at 48 hours, and subsequent marked decline started from $72-96$ hours post-LPS challenge (Figure 1A). The number of neutrophils, macrophages, lymphocytes, and total cells in BALF was quantified at 48 hours post-LPS instillation, corresponding to 24 hours post-SPIO nanoparticle instillation for the four different groups: control, SPIO, LPS, and LPS-SPIO (Figure 1B). The numbers of neutrophils $\left(2.34 \times 10^{6} \pm 0.31 \times 10^{6}\right)$ and macrophages $\left(1.74 \times 10^{6} \pm 0.19 \times 10^{6}\right)$ were found to considerably increase in the BALF compared with lymphocytes $\left(0.15 \times 10^{6} \pm 0.07 \times 10^{6}\right)(P<0.05)$. 


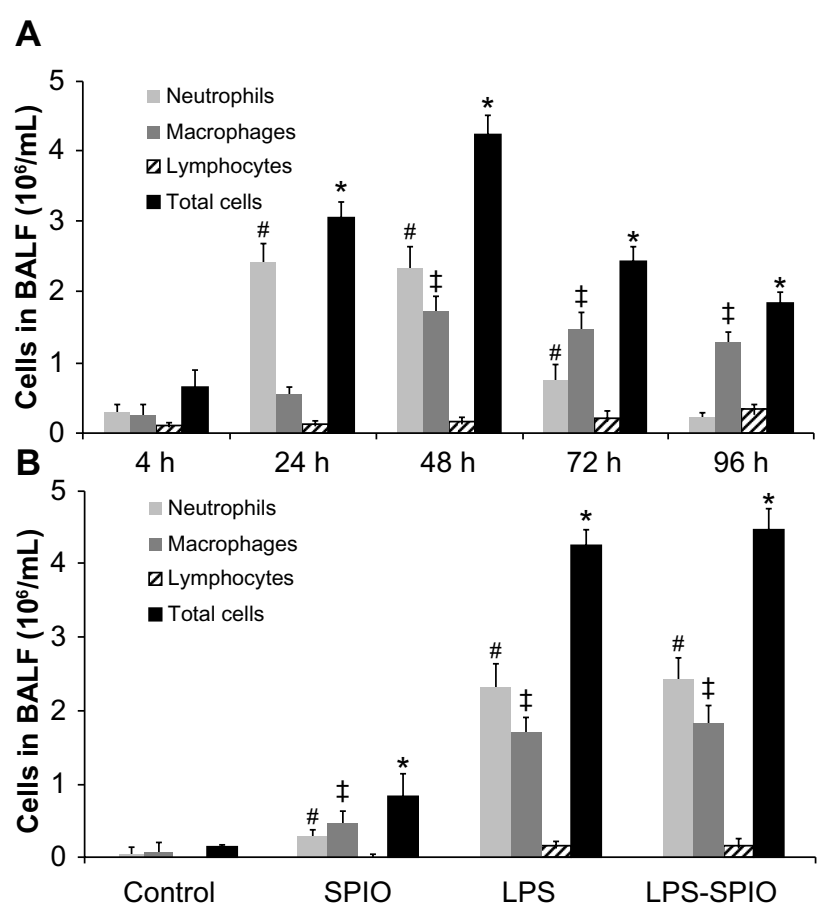

Figure I Number of neutrophils, macrophages, lymphocytes, and total cells in BALF of (A) mice intrapulmonary exposed to LPS at different investigation time points, and (B) control, SPIO, LPS, and LPS-SPIO mice at 48 hours post-LPS challenge.

Notes: Data expressed as mean \pm standard deviation, $\mathrm{n}=6$ per group. \#, ₹, *, $P<0.05$.

Abbreviations: BALF, bronchoalveolar lavage fluid; LPS, lipopolysaccharide; SPIO, superparamagnetic iron oxide; h, hours.

Remarkably, while LPS significantly increased the numbers of inflammatory cells, SPIO nanoparticles were found to cause only a minor increase in both macrophages and neutrophils 24 hours post-instillation compared with control group $(P<0.05)$.
To investigate the polarization state of AMs after lung administration of either SPIO, LPS, or both LPS and SPIO, the levels of IL-12 and CXCL-10 as markers of M1 macrophages and the levels of IL-4 and CCL-22 as markers of M2 macrophages was assessed using ELISA.

At 48-hours post-exposure, the levels of ILs (IL-12 and IL-4) and chemokines (CXCL-10 and CCL-22) were found to be higher than control group in both LPS and LPS-SPIO groups; however, only the level of CXCL-10 was found to statistically increase $(P<0.05)$ (Figure 2$)$.

To further evaluate the effect of LPS and/or SPIO instillation in the lung, the relative gene expression of NOS-2, CXCL-10, and TNF- $\alpha$ as markers of M1 macrophages and CCL-17, CCL-22, Arg1, and IL-10 as markers of M2 macrophages in SPIO, LPS, and LPS-SPIO mice compared with control mice was performed. A considerable increase in all gene markers was found 48 hours following LPS intrapulmonary exposition, with a 145 -fold increase observed for CXCL-10, a 78-fold increase for TNF- $\alpha$, a 28-fold increase for CCL-17, and a 12-fold increase for IL-10, compared with control group (Figure 3). However, a limited increase in gene expression was observed in the SPIO group, with a maximum of 10-fold increase observed for CXCL-10, and 1.8, 3.0, 3.3, and 4.4-fold increases observed for Arg1, IL-10, TNF- $\alpha$, and CCL-17, respectively.

Furthermore, to differentiate the macrophage subpopulations based on their specific surface membrane receptor expression, flow cytometry analysis was performed on AMs obtained from the BALF of the different groups, which were
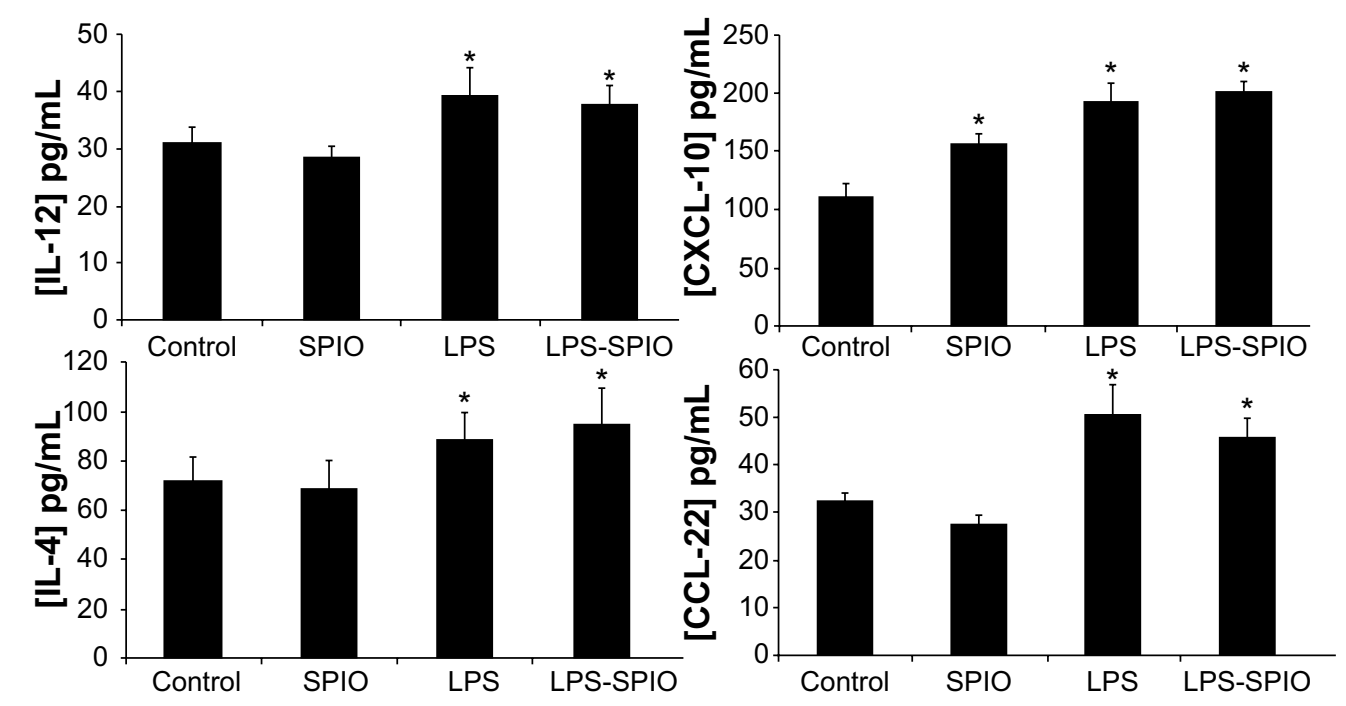

Figure 2 Measurement of IL-12 and CXCL-10 levels as markers of MI macrophages and IL-4 and CCL-22 levels as markers of M2 macrophages in BALF of control, SPIO, LPS, and LPS-SPIO groups at 48 hours post-LPS challenge.

Notes: Data expressed as mean \pm standard deviation, $\mathrm{n}=6$ per group; $* \mathrm{P}<0.05$.

Abbreviations: BALF, bronchoalveolar lavage fluid; IL, interleukin; LPS, lipopolysaccharide; SPIO, superparamagnetic iron oxide; CXCL-I0, chemokine (C-X-C motif) ligand 10; CCL-22, chemokine (C-C motif) ligand 22. 


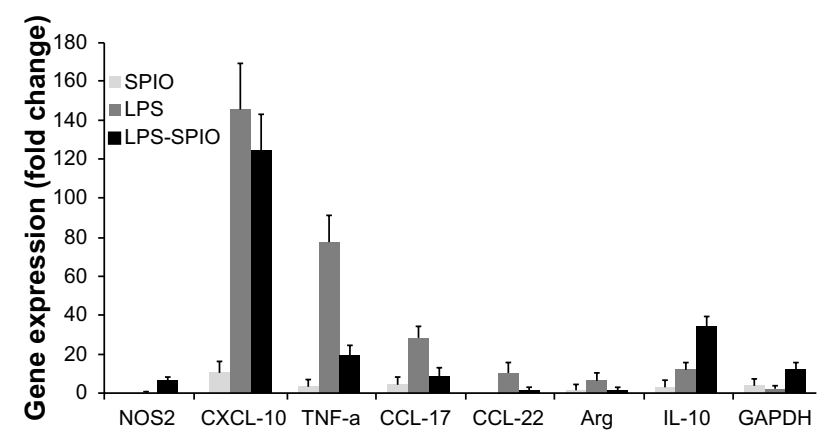

Figure 3 Relative gene expression of NOS-2, CXCL-10, and TNF as markers of MI macrophages and CCL-17, CCL-22, ArgI, and IL- 10 as markers of M2 macrophages in SPIO, LPS, and LPS-SPIO mice compared with control mice. GAPDH was analyzed as an internal control.

Note: Data expressed as mean \pm standard deviation, $n=6$ per group.

Abbreviations: Arg, arginase; GAPDH, glyceraldehyde 3-phosphate dehydrogenase; IL, interleukin; LPS, lipopolysaccharide; NOS, nitric oxide synthase; SPIO, superparamagnetic iron oxide; TNF, tumor necrosis factor; CXCL-10, chemokine (C-X-C motif) ligand I0; CCL-I7, chemokine (C-C motif) ligand I7.

allowed to adhere in cell culture flask for 2 hours to obtain only adherent cells.

Adherent cells were found to be CD11b positive in all groups, indicating that macrophages were obtained. Following LPS exposure, macrophages exhibited an over-expression of both CD86 and CD206, with $41.9 \% \pm 3.7 \%$ and $34.1 \% \pm 3.1 \%$, respectively, in the LPS group compared with $12.3 \% \pm 2.6 \%$ and $22.1 \% \pm 2.2 \%$, respectively, in the control group (Figure 4 ). The percentage of cells expressing CD86 was significantly increased $(P<0.05)$ with SPIO, LPS, and LPS-SPIO, and cells expressing CD206 were significantly increased with LPS and LPS-SPIO. In addition, intrapulmonary instillation of SPIO statistically increased $(P<0.05)$ the expression of CD86 $(28.7 \% \pm 2.9 \%$ in the SPIO group compared with $12.3 \% \pm 2.6 \%$ in the control group), while the increase in CD206 $(28.2 \% \pm 1.9 \%$ in the SPIO group compared with $22.1 \%$ in the control group) was not statistically significant $(P>0.05)$. Conversely, CD197 and CD150 revealed a high overexpression in all the different groups, with no significant variation after treatment

\section{Physical characterization of antibody- conjugated SPIO nanoparticles}

CD86- and CD206-conjugated SPIO nanoparticles were then developed to specifically target M1- and M2-macrophage subpopulations, respectively, in the lung of

A
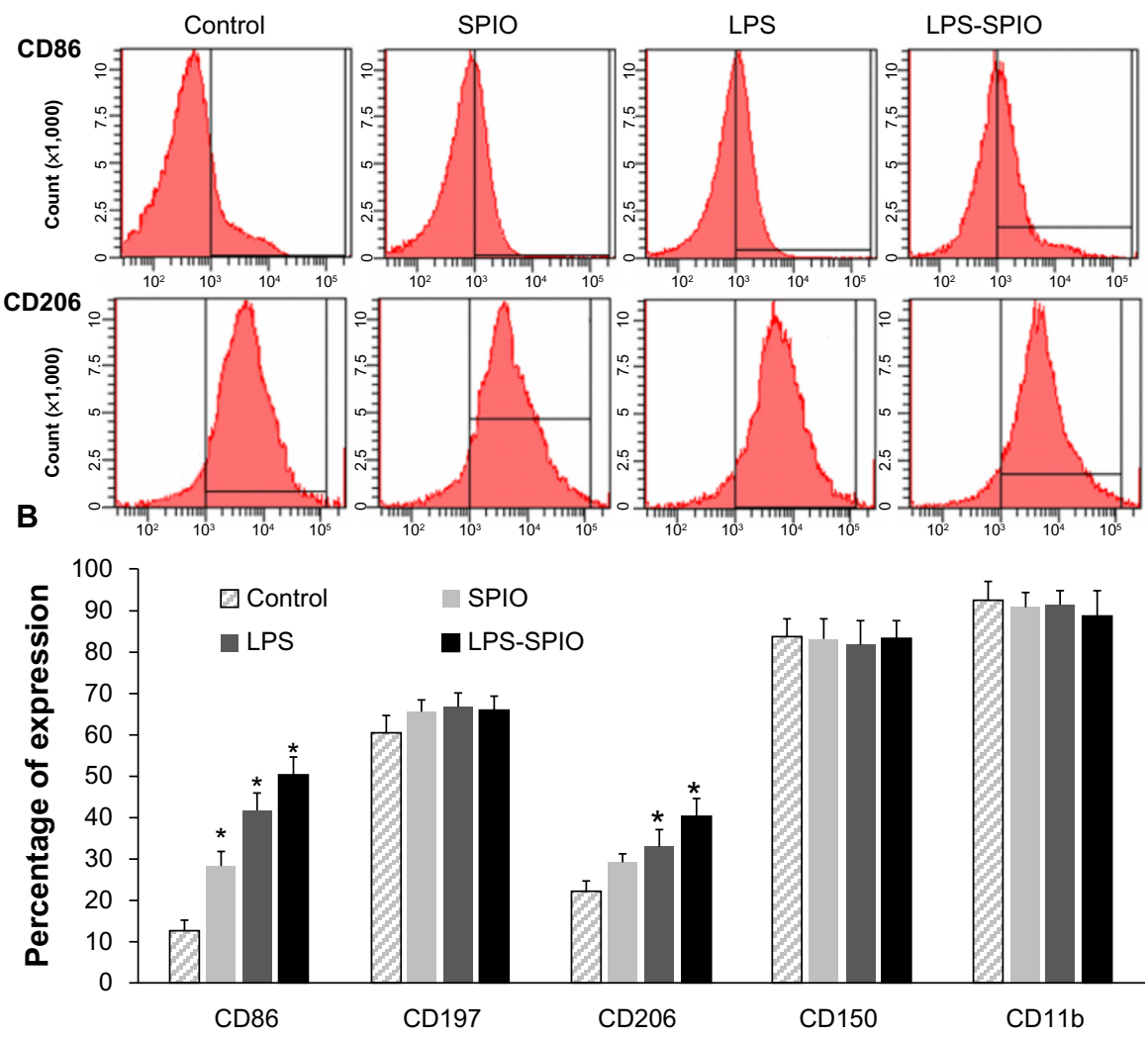

Figure 4 Flow cytometry analysis of alveolar macrophages issued from the BALF of control, SPIO, LPS, and LPS-SPIO groups. (A) Representative histogram of CD86 (higher row) and CD206 (lower row) expression. (B) Percentage of cells expressing CD86, CDI97, CD206, CDI50, and CDIIb.

Notes: Error bars are standard deviation of triplicates. $* P<0.05$.

Abbreviations: BALF, bronchoalveolar lavage fluid; LPS, lipopolysaccharide; SPIO, superparamagnetic iron oxide; CD, cluster of differentiation. 
the LPS-induced COPD model to enable their noninvasive imaging using MRI.

Measurement of the hydrodynamic size revealed that the mean nanoparticle diameter increased after conjugation of CD86 and CD206 antibodies to $133.7 \mathrm{~nm}$ and $123.0 \mathrm{~nm}$, respectively, whereas the size of non-conjugated SPIO nanoparticles was $82.7 \mathrm{~nm}$.

Quantification of protein concentration using BCA assay showed that the amount of bound antibody was $15 \mu \mathrm{g}$ and $29 \mu \mathrm{g}$ per mg of iron oxide for CD86- and CD206-conjugated SPIO nanoparticles, respectively.

Measurement of $\mathrm{r} 1, \mathrm{r} 2$, and $\mathrm{r} 2 *$ relaxivity values at $4.7 \mathrm{~T}$ exhibited very high $\mathrm{r} 2$ and $\mathrm{r} 2 *$ values $(229.48 \pm 3.44$ and $264.52 \pm 4.16 \mathrm{mM}^{-1} \cdot \mathrm{s}^{-1}$ ) for SPIO nanoparticles that slightly decreased after conjugation with either CD86 (201.48 \pm 5.89 and $234.24 \pm 6.16 \mathrm{mM}^{-1} \cdot \mathrm{s}^{-1}$ for $\mathrm{r} 2$ and $\mathrm{r} 2 *$, respectively) or CD206 (209.84 \pm 7.51 and 229.97 $\pm 6.27 \mathrm{mM}^{-1} \cdot \mathrm{s}^{-1}$ for $\mathrm{r} 2$ and $\mathrm{r} 2 *$, respectively) antibodies. For all samples, r1 values were found negligible $\left(0.06 \pm 0.01 \mathrm{mM}^{-1} \cdot \mathrm{s}^{-1}\right)$.

\section{Noninvasive targeting and imaging of AM subpopulations}

Noninvasive MRI performed at 48 hours post-LPS intrapulmonary instillation using UTE sequence enabled successful detection of hyperintensity regions related to edema in the lung. These regions were homogenously distributed in the different lobes. Quantitatively, the number of hyperintensity pixels per slice was found to increase to $3897 \pm 495$ in 48 hours
post-LPS group, compared with $1975 \pm 398$ in control group (Figure 5).

Furthermore, the possibility of using a very short TE enabled obtaining enough MRI signal in lung parenchyma, which has always been limited by conventional MRI sequences. This protocol has offered the possibility of successful detection of void signal dots related to antibodyconjugated iron oxide nanoparticles in the lung. The number of hyperintensity pixels was therefore found to decrease significantly $(P<0.05)$ following intrapulmonary administration of either CD86- or CD206-conjugated SPIO nanoparticles to $2910 \pm 356$ and $2845 \pm 394$, respectively. Interestingly, these antibody-conjugated magnetic nanoparticles were found to co-localize with the inflammatory regions in the lungs (red arrows in Figure 5), while non-conjugated SPIO nanoparticles were not found to confine to the inflammatory regions.

\section{Characterization of polarization profile of targeted AMs}

To confirm the MRI readouts and assess the polarization state of targeted AM subpopulations, flow cytometry and immunohistochemistry analysis following the MRI protocol were performed.

Flow cytometry analysis on targeted macrophage subpopulations sorted from total cellular extracts of mice lungs showed a statistically significant increase in the expression of CD86 (50.7\% $\pm 5.4 \%)$ in the eluted bound cell fractions (ie, M1 targeted macrophages) of 48-hour post-LPS
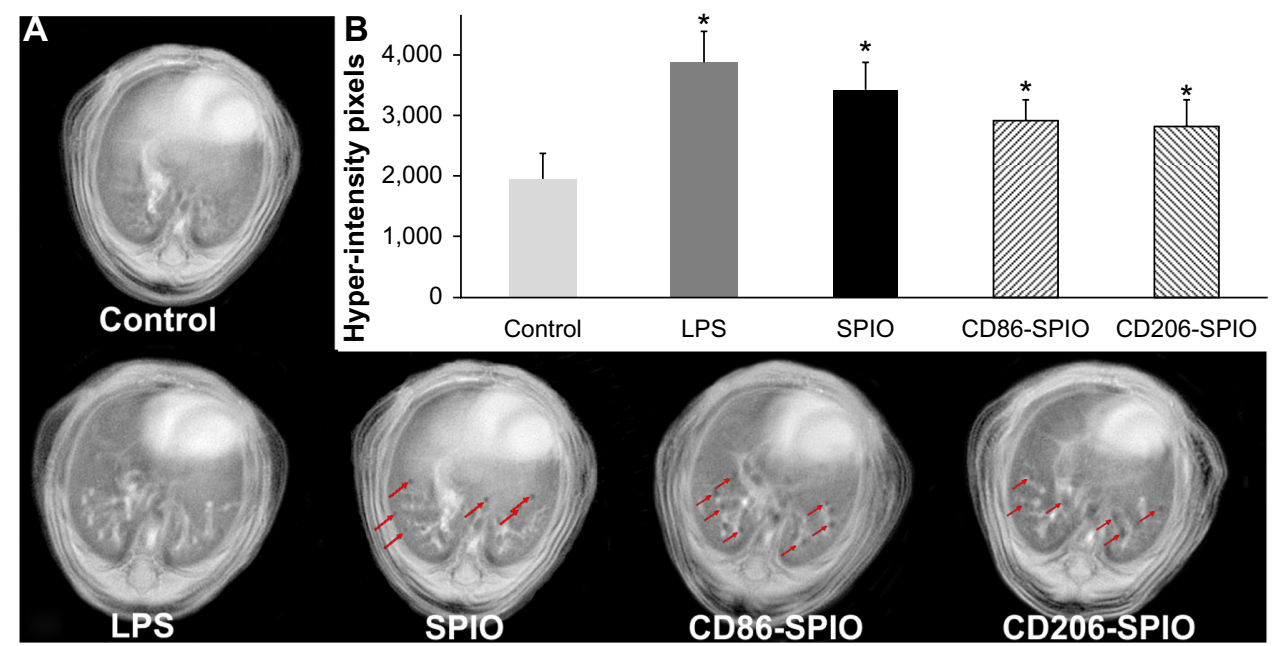

Figure 5 (A) Representative magnetic resonance images acquired on a 4.7T magnet, using a radial ultra-short echo time sequence of a control, LPS (48 hours post-LPS induction), SPIO, CD86-SPIO, and CD206-SPIO groups (2 hours post-intrapulmonary administration of either non-conjugated, CD86-conjugated, or CD206-conjugated SPIO nanoparticles) of 48 hours post-LPS-induced COPD in mice.

Notes: (A) Red arrows highlight the presence of void signal dots related to the presence of the nanoparticles, which were found to co-localize with inflammatory regions with antibody-conjugation SPIO nanoparticles. (B) Quantification of hyperintensity pixels, averaged on a set of ten slices, with $0.5 \mathrm{~mm}$ inter-slices which were positioned at the same position in lung parenchyma of the different groups. Data expressed as mean \pm standard deviation, $n=6$ per group. $* P<0.05$.

Abbreviations: LPS, lipopolysaccharide; SPIO, superparamagnetic iron oxide; CD, cluster of differentiation. 
mice instilled with CD86-conjugated SPIO nanoparticles (LPS + CD86-SPIO (+)), whereas, an overexpression of CD206 (58.7\% $\pm 3.6 \%)$ was detected in the eluted bound cell fractions (ie, M2 targeted macrophages) of 48-hour post-LPS mice instilled with CD206-conjugated SPIO nanoparticles (LPS + CD206-SPIO $(+))(P<0.05)$. No statistically significant variation was observed in the other groups (Figure 6).

The successful targeting of M1 and M2 macrophages in LPS-induced COPD lungs using antibody-conjugated SPIO nanoparticles was also confirmed using immunohistochemistry analysis. While the intrapulmonary instillation of the SPIO nanoparticles used under the current experimental conditions (ie, dose, chemical composition, and exposure method) was found to minimally alter the number of macrophages in the lung, LPS exposition was found to highly increase the level of F4/80 as a universal marker of macrophages, and equally overexpress both inducible NOS (iNOS) and Arg1 as a marker of M1 and M2 macrophages (Figure 7).
Following intrapulmonary administration of either CD86or CD206-SPIO nanoparticles, a high number of blue dots were detected using Perl's staining (black arrows, Figure 7) and found to be co-localized with iNOS and Arg1 markers, indicating successful targeting of the different AM subpopulations, while no co-localization was observed following administration of non-conjugated SPIO nanoparticles. No significant variation in the expression levels of iNOS and Arg1 was observed following the instillation of the different SPIO nanoparticles.

\section{Discussion}

In this study, a noninvasive imaging protocol coupled with the use of biocompatible antibody-conjugated iron oxide nanoparticles was developed to enable specific targeting and imaging of a particular macrophage subset in COPD. Noninvasive tracking of a single-cell population in inflammatory diseases is required for both diagnostic purposes and therapeutic applications. ${ }^{22,23}$ To validate our approach,

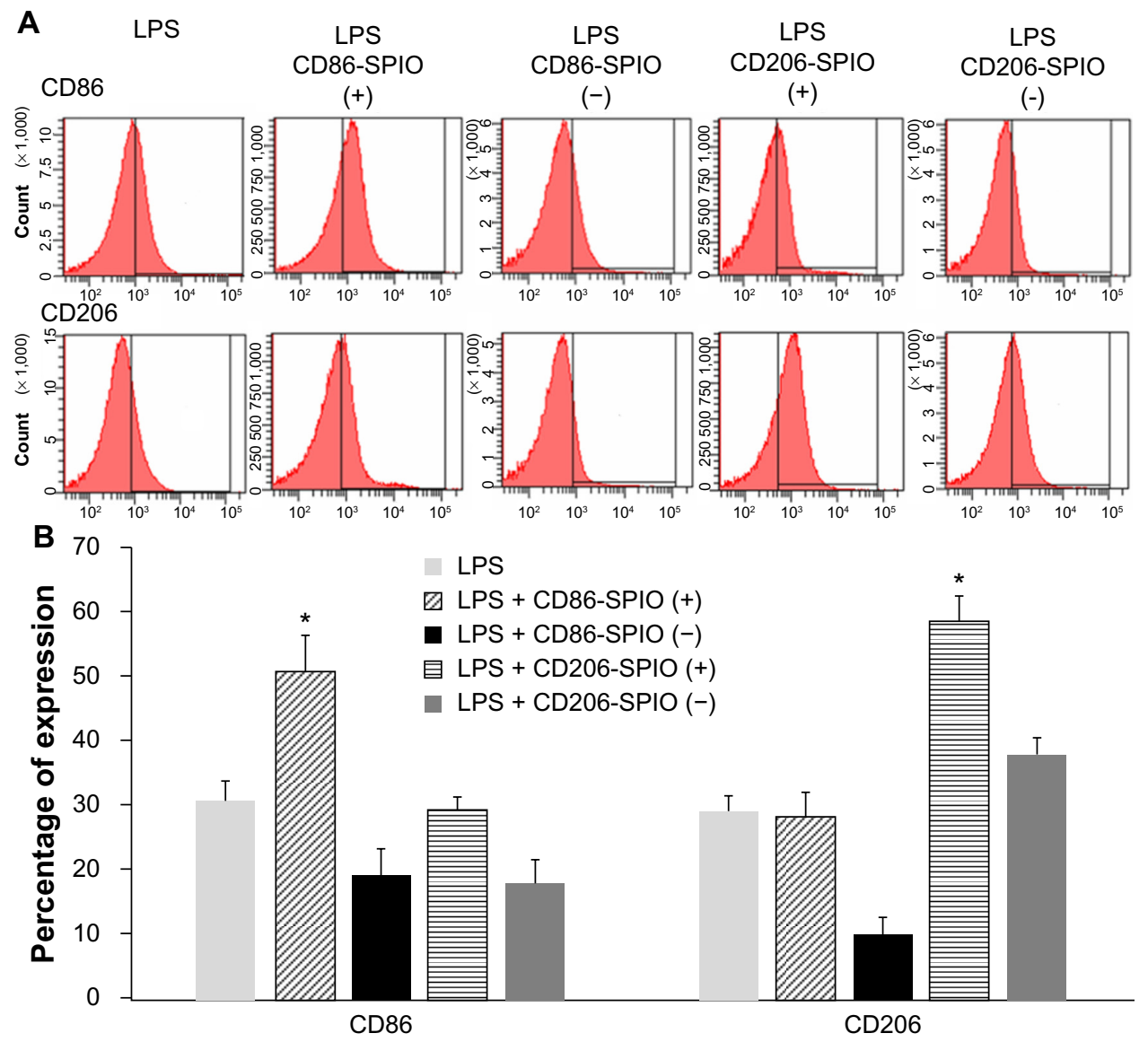

Figure 6 Flow cytometry analysis of targeted macrophage subpopulations sorted using magnet attraction from total cellular extracts of 48 hours post-LPS-induced COPD mice lungs before and after intrapulmonary instillation of either CD86-conjugated (LPS + CD86-SPIO) or CD206-conjugated (LPS + CD206-SPIO) SPIO nanoparticles. (A) Representative histogram of CD86 (high row) and CD206 (lower row) expression. (B) Percentage of cells expressing CD8 and CD206 in the different groups. Notes: $(+)$ and $(-)$ refer to eluted bound or unbound cell fractions, respectively. Error bars are standard deviation of triplicates. $* P<0.05$.

Abbreviations: COPD, chronic obstructive pulmonary disease; LPS, lipopolysaccharide; SPIO, superparamagnetic iron oxide; CD, cluster of differentiation. 


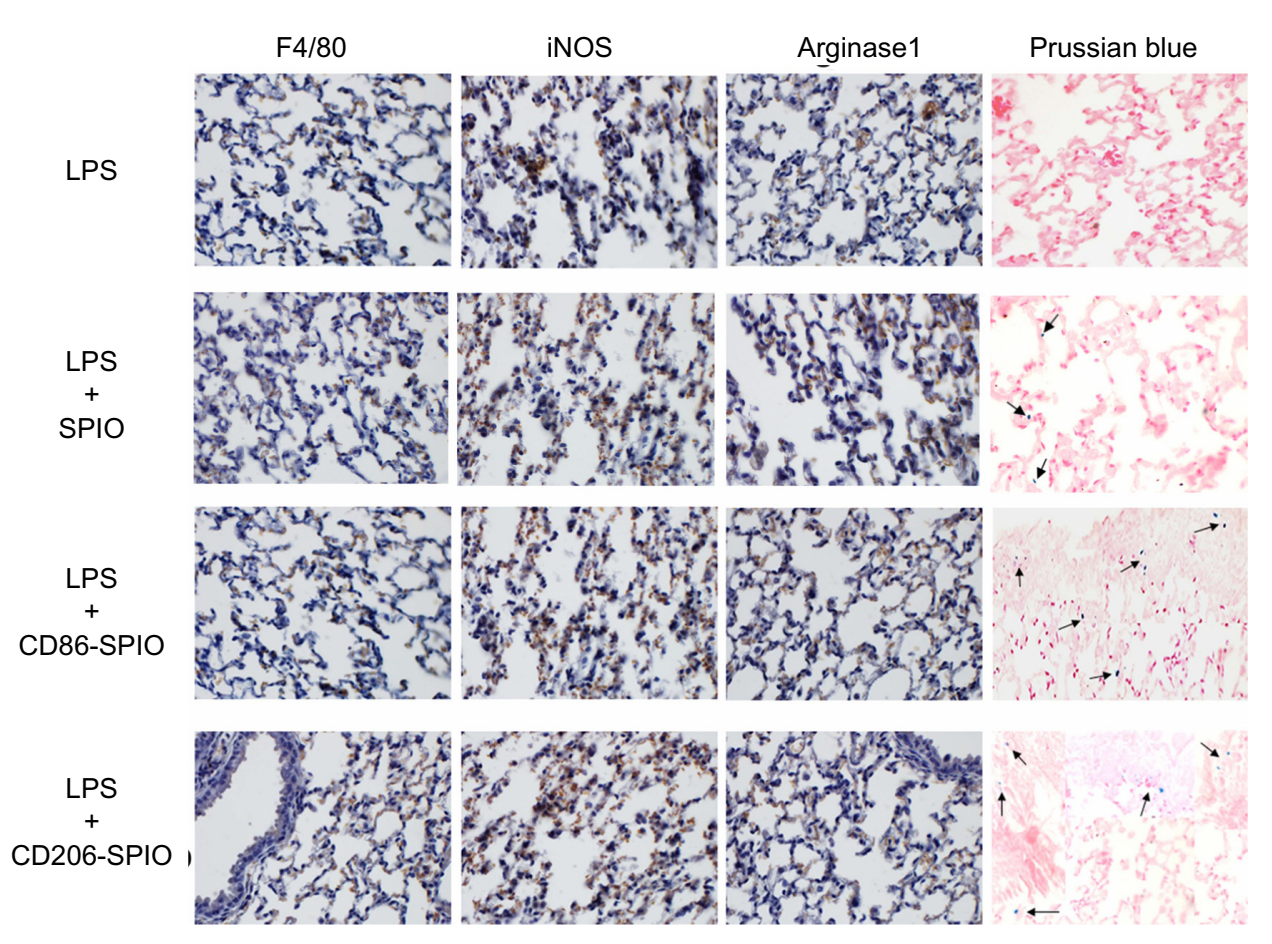

Figure 7 Immunohistochemistry analysis of F4/80 (universal marker of macrophages), iNOS (marker for MI macrophages), Arginasel (marker for M2 macrophages), and Prussian blue (marker for iron oxide) staining in 48 hours post-LPS-induced COPD lung before and after intrapulmonary administration of either SPIO, CD86-SPIO, or CD206-SPIO nanoparticles.

Note: Final original magnification, $\times 400$.

Abbreviations: COPD, chronic obstructive pulmonary disease; iNOS, inducible nitric oxide synthase; LPS, lipopolysaccharide; SPIO, superparamagnetic iron oxide; CD, cluster of differentiation.

the effect of intrapulmonary administration of iron oxide nanoparticles on the polarization profile of AMs was first investigated. Then, a specific biomarker for each macrophage subpopulation was selected for conjugation with biocompatible SPIO nanoparticles. This complex has enabled, for the first time, a noninvasive detection of a specific subpopulation of macrophages in the lung of LPS-induced COPD model using a free-breathing MRI protocol.

Extensively used in preclinical investigations, the intrapulmonary exposure of the bacterial endotoxin LPS triggers the production of inflammatory mediators, which results in enhanced macrophage phagocytic and cytotoxic activity and reproduces the pathophysiological features that resemble COPD. ${ }^{24-27}$ While the induction of lung inflammation in response to LPS is well documented, the inflammatory process has been shown to depend on various factors such as animal strain, route and dose of LPS exposition, and bacterial strain used (ie, Escherichia coli or Pseudomonas aeruginosa), which might affect its relevance as a model of COPD. ${ }^{28,29}$ Therefore, a full characterization of the pulmonary inflammatory process has to be performed under the experimental conditions used.

Similar to what has been reported in the different studies, ${ }^{29-31}$ the peak inflammatory process was observed at
48 hours post-LPS challenge, with neutrophils and macrophages forming more than $95 \%$ of total inflammatory cells in BALF. However, while LPS significantly increased the number of inflammatory cells, the intrapulmonary administration of PEGylated dextran-coated iron oxide nanoparticles $\left([\mathrm{Fe}]=4 \mathrm{mM} ; \mathrm{V}=100 \mu \mathrm{L}\right.$ ) using the MicroSprayer ${ }^{\circledR}$ aerosolizer were found to only slightly increase macrophages and neutrophils at 24 hours post-administration, suggesting the safety of the SPIO nanoparticles used.

While different studies have reported the safety of different formulations of engineered iron oxide nanoparticles for biomedical applications, ${ }^{32}$ and some iron oxide nanoparticles have obtained US FDA approval as MRI contrast agents, there is still some discrepancy in the literature about the safety of these nanomaterials for pulmonary biomedical applications. Therefore, a long-term assessment of the chronic safety of iron oxide nanoparticles is required for safe and effective respiratory medical applications based on nanotechnology.

To investigate the polarization state of AMs after intrapulmonary instillation of either SPIO and/or LPS, cytokine-release measurement using ELISA, relative gene expression using RT-PCR, and surface membrane receptor expression using flow cytometry analysis were performed on the BALF of mice lungs. While LPS significantly increased 
the levels of all M1 (ie, IL-12, NOS-2, CXCL-10, TNF- $\alpha$, and CD86) and M2 (ie, IL-4, IL-10, CCL-17, CCL-22, Arg1, and CD206) biomarkers, the intrapulmonary instillation of PEGylated dextran-coated SPIO nanoparticles was found to slightly elevate the levels of CXCL-10 in ELISA, accompanied with an increase in its relative gene expression along with an overexpression of CD86, which is a marker of M1 polarization.

Significant elevations of IL-12, IL-4, CXCL-10, and CCL-22 were observed with both LPS and LPS-SPIO treatment $(P<0.05)$. SPIO induced significant elevations in CXCL-10 but not in other cytokines and ILs (Figure 2). Gene expression analyses also showed 10 -fold elevations in CXCL-10 with SPIO, in contrast to either LPS or LPS-SPIO groups which showed expression levels of more than 100-fold increase. However, it has been observed that there was no decrease in the viability and biocompatibility of cells when treated with SPIO. As previously reported, ${ }^{33}$ although the levels of CXCL-10 or certain chemokines are elevated, iron oxide nanoparticles do not cause significant changes in the immune functions of cells.

The overexpression of both M1 and M2 markers following LPS lung administration was previously reported in several studies to orchestrate the pathophysiology of inflammatory process during COPD by activating either a pro-inflammatory M1 or immunomodulatory M2 profiles, depending on the environmental conditions of the inflammatory process. ${ }^{4,29,34}$ However, the increase in the expression of M1 markers (CXCL-10 and CD86) following SPIO nanoparticle administration may indicate that macrophages have recognized these nanoparticles as foreign, thus functioning to phagocytize them by activating their antigen-presenting function.

It must be reemphasized that although the gene expression of some markers (eg, CXCL-10, CCL-7, and TNF- $\alpha$ ) was higher compared with control, the nanoparticles used in this study can be assumed biocompatible and safe for in vivo preclinical applications. However, complementary large-scale and long-term studies are required to conclusively establish this aspect for prospective clinical applications.

After assessing the biocompatibility of the PEGylated dextran-coated iron oxide nanoparticles and the effect of intrapulmonary administration of SPIO and/or LPS on the polarization profile of AM subpopulations, monoclonal antibodies raised against either CD86 or CD206 were conjugated to the iron oxide nanoparticles to specifically target M1 or M2 macrophage subpopulations, respectively, in the lung of an LPS-induced COPD model and enable their noninvasive detection using MRI.
Targeted magnetic nanoprobes have the potential to become highly selective imaging tools. The feasibility of successfully conjugating antibody molecules to SPIO nanoparticles was confirmed by the BCA assay, which revealed $15 \mu \mathrm{g}$ of CD86 and $29 \mu \mathrm{g}$ of CD206 antibodies per mg of SPIO nanoparticles, as well as the measurements of the hydrodynamic size, which indicated an increase in the nanoprobe size from $82.7 \mathrm{~nm}$ for non-conjugated to 133.7 and $123.0 \mathrm{~nm}$ for CD86 and CD206 antibody-conjugated SPIO nanoparticles, respectively. The size of administered nanoprobes to the lung is a key factor that regulates the delivery of therapeutics and contrast agents through the pulmonary route and particularly affects the rate of engulfment by AMs. Therefore, nanoparticles in the range of $100 \mathrm{~nm}$ were preferred for pulmonary administration in this study, as they should be small enough to pass through the conducting airways in the lung but not too small that they fail to deposit and are exhaled again. ${ }^{35}$

On the other hand, magnetic nanoparticles with increased $\mathrm{r} 2$ or $\mathrm{r} 2 *$ transverse relaxivities are needed to enhance their contrast in MRI images and therefore their efficient detection, especially for pulmonary applications with very limited MRI signal in lung parenchyma.

The PEGylated dextran-coated iron oxide nanoparticles with an iron oxide crystallite diameter of 10-13 $\mathrm{nm}$ and a total size of $\sim 100 \mathrm{~nm}$ were found to have very high $\mathrm{r} 2$ and $\mathrm{r} 2 *$ relaxivities $\left(229.48 \pm 3.44\right.$ and $\left.264.52 \pm 4.16 \mathrm{mM}^{-1} \cdot \mathrm{s}^{-1}\right)$ for non-conjugated SPIO nanoparticles, which slightly decreased after conjugation with either CD86 (201.48 \pm 5.89 and $234.24 \pm 6.16 \mathrm{mM}^{-1} \cdot \mathrm{s}^{-1}$ for $\mathrm{r} 2$ and $\mathrm{r} 2 *$, respectively) or

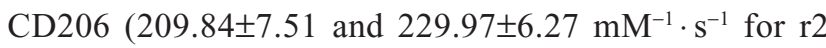
and $\mathrm{r} 2 *$, respectively) antibodies. This chemical composition was reported to facilitate the exchange and diffusion of water molecules to the magnetic core of the magnetic nanoparticles, ${ }^{36,37}$ and these relaxivity values were found to be much higher than the clinically approved MRI contrast agents Feridex $^{\circledR}$ IV (Berlex Laboratories, Wayne, NJ, USA) and Resovist ${ }^{\circledR}$ (Bayer Healthcare, Berlin, Germany) that were reported in the literature. ${ }^{38,39}$

A free-breathing MRI protocol was then optimized to enable the simultaneous visualization of inflammation progression in the lung of LPS-induced COPD animal model and the noninvasive detection of M1 or M2 macrophage subpopulations, which were specifically targeted with the developed antibody-conjugated nanoprobes.

The possibility of inflammation detection in the lung following LPS exposition using a free-breathing MRI protocol was first introduced by Beckmann et $\mathrm{al}^{31}$ using a gradient echo sequence with a high number of excitations (ie, Nex $=60) .{ }^{40}$ 
The absence of a lung parenchymal signal in combination with a background free of artifacts has provided a high contrast-to-noise ratio for the detection of signals in response to LPS challenge. However, this protocol was limited for the detection of iron oxide-based nanoparticles, which induce a negative contrast in MRI images.

With the recent introduction of UTE pulse sequence in $\mathrm{MRI}^{13}$ and its validation as an approach particularly valuable in imaging lung tissue, which is characterized by very short $\mathrm{T} 2 *$ relaxation time, ${ }^{41}$ Conti et $\mathrm{al}^{42}$ showed that UTE sequence is able to better detect inflammatory regions in lung parenchyma which were, in some slices, undetectable by standard gradient echo sequence.

By using a very short TE (ie, TE $=0.4 \mathrm{~ms}$ ), UTE sequence enabled enough MRI signal in the parenchyma of lung and therefore offers the possibility of sensitively detecting magnetic nanoparticles in the lung. In the current study, following CD86- and CD206-conjugated iron oxide nanoparticles, void signal dots related to targeting either M1 or M2 macrophages were successfully detected in the lung of COPD model (ie, $25 \%$ decrease in hyperintensity pixels) and were found to co-localize with the inflammatory regions induced by LPS challenge.

The successful recruitment of antibody-conjugated SPIO nanoparticles by macrophage subpopulations in the lung following their intrapulmonary instillation was thus confirmed using flow cytometry and immunohistochemistry analyses, which were also performed to characterize the polarization profile of targeted AMs.

Following magnetic sorting of total cellular extracts of mice lungs, an overexpression of CD86 and CD206 was detected in the eluted bound fractions of CD86- and CD206conjugated SPIO nanoparticles, respectively, confirming the selective targeting of M1 and M2 macrophage subpopulations, respectively. Besides, no variation in the expression of CD86 and CD206 was observed in the other groups.

In accordance with these observations, a co-localization of either CD86- or CD206-conjugated iron oxide nanoparticles revealed through positive Prussian blue staining was detected with either iNOS M1-specific or Arg1 M2-specific marker in LPS-induced lung using immunohistochemistry analysis. Furthermore, no variation in the expression levels of iNOS and Arg1, which were highly expressed following LPS pulmonary challenge, was observed following the administration of the macrophage-targeted nanoprobes.

Although it becomes evident that AMs are extremely versatile cells and can switch their polarization profile depending on the environmental conditions, a balanced proportion of macrophage subpopulations was found to predominate even though a continuum switch in their polarization might occur. Accordingly, this study provides preliminary evidence for the presence of a balanced number of M1 and M2 macrophages at early stages of LPS-induced inflammation, which we believe may continuously change their polarization state during the time course of inflammation. However, further confirmatory studies are needed to conclusively establish this phenomenon.

\section{Conclusion}

In conclusion, AM polarization was extensively characterized after LPS and/or SPIO intrapulmonary administration. Specific biomarkers targeted to either M1 or M2 macrophage subpopulations were identified. Biocompatible antibodyconjugated iron oxide nanoparticles have thus been reported in the current paper as a novel approach to specifically target one subpopulation of macrophages in the lung of a COPD animal model and enable their noninvasive tracking using a free-breathing MRI protocol. Flow cytometry and immunohistochemistry analysis confirmed MRI readouts and suggested a balance in the number of M1 and M2 macrophages during the inflammatory process and after pulmonary administration of the nanoprobes that can serve as diagnostic and therapeutic agents.

\section{Acknowledgments}

This work was supported by NPST Program, King Saud University; Project Number 11-MED1773. We thank Cordula Gruttner from Micromod Partikeltechnologie GmbH for help in nanoparticle conjugation and Emmanuelle Canet-Soulas from the University of Lyon for helpful discussion.

\section{Disclosure}

The authors report no conflicts of interest in this work.

\section{References}

1. Smith I, Schallenberg, M. Occurrence of the agricultural nitrification inhibitor, dicyandiamide, in surface waters and its effects on nitrogen dynamics in an experimental aquatic system. Agric Ecosyst Environ. 2013;164:23-31.

2. Barnes P. Alveolar macrophages as orchestrators of COPD. COPD. 2004;1(1):59-70.

3. Alber A, Howie SE, Wallace WA, Hirani N. The role of macrophages in healing the wounded lung. Int J Exp Pathol. 2012;93(4):243-251.

4. Herold S, Mayer K, Lohmeyer J. Acute lung injury: how macrophages orchestrate resolution of inflammation and tissue repair. Front Immunol. 2011;2:65.

5. Duan M, Li WC, Vlahos R, Maxwell MJ, Anderson GP, Hibbs ML. Distinct macrophage subpopulations characterize acute infection and chronic inflammatory lung disease. J Immunol. 2012;189(2): 946-955.

6. Weissleder R. Molecular imaging in cancer. Science. 2006;312(5777): $1168-1171$. 
7. McCarthy JR, Weissleder R. Multifunctional magnetic nanoparticles for targeted imaging and therapy. Adv Drug Deliv Rev. 2008;60(11): 1241-1251.

8. Oghabian MA, Farahbakhsh NM. Potential use of nanoparticle based contrast agents in MRI: a molecular imaging perspective. $J$ Biomed Nanotechnol. 2010;6(3):203-213.

9. Tassa C, Shaw SY, Weissleder R. Dextran-coated iron oxide nanoparticles: a versatile platform for targeted molecular imaging, molecular diagnostics, and therapy. Acc Chem Res. 2011;44(10):842-852.

10. Jokerst JV, Gambhir SS. Molecular imaging with theranostic nanoparticles. Acc Chem Res. 2011;44(10):1050-1060.

11. Modo MMJ, Bulte JWM. Molecular and Cellular MR Imaging. CRC Press; 2007.

12. Beckmann N, Cannet C, Babin AL, et al. In vivo visualization of macrophage infiltration and activity in inflammation using magnetic resonance imaging. Wiley Interdiscip Rev Nanomed Nanobiotechnol. 2009;1(3):272-298.

13. Tyler DJ, Robson MD, Henkelman RM, Young IR, Bydder GM. Magnetic resonance imaging with ultrashort TE (UTE) PULSE sequences: technical considerations. J Magn Reson Imaging. 2007;25(2):279-289.

14. Zurek M, Boyer L, Caramelle P, Boczkowski J, Cremillieux Y. Longitudinal and noninvasive assessment of emphysema evolution in a murine model using proton MRI. Magn Reson Med. 2012;68(3): 898-904.

15. Al Faraj A, Luciani N, Kolosnjaj-Tabi J, et al. Real-time high-resolution magnetic resonance tracking of macrophage subpopulations in a murine inflammation model: a pilot study with a commercially available cryogenic probe. Contrast Media Mol Imaging. 2013;8(2):193-203.

16. Al Faraj A. Preferential magnetic nanoparticle uptake by bone marrow derived macrophages sub-populations: effect of surface coating on polarization, toxicity, and in vivo MRI detection. J Nanopart Res. 2013;15(7):1-13.

17. Martinez FO, Helming L, Gordon S. Alternative activation of macrophages: an immunologic functional perspective. Annu Rev Immunol. 2009;27:451-483

18. Shahbazi-Gahrouei D, Abdolahi M, Zarkesh-Esfahani SH, Laurent S, Sermeus C, Gruettner C. Functionalized magnetic nanoparticles for the detection and quantitative analysis of cell surface antigen. Biomed Res Int. 2013;2013:349408.

19. Rimkus G, Bremer-Streck S, Grüttner C, Kaiser WA, Hilger I. Can we accurately quantify nanoparticle associated proteins when constructing high-affinity MRI molecular imaging probes? Contrast Media Mol Imaging. 2011;6(3):119-125.

20. Al Faraj A, Bessaad A, Cieslar K, Lacroix G, Canet-Soulas E, Cremillieux Y. Long-term follow-up of lung biodistribution and effect of instilled SWCNTs using multiscale imaging techniques. Nanotechnology. 2010;21(17):175103.

21. Vazquez-Tello A, Halwani R, Li R, et al. IL-17A and IL-17F expression in B lymphocytes. Int Arch Allergy Immunol. 2012;157(4):406-416.

22. Richards JM, Shaw CA, Lang NN, et al. In vivo mononuclear cell tracking using superparamagnetic particles of iron oxide: feasibility and safety in humans. Circ Cardiovasc Imaging. 2012;5(4):509-517.

23. Arbab AS, Janic B, Haller J, Pawelczyk E, Liu W, Frank JA. In vivo cellular imaging for translational medical research. Curr Med Imaging Rev. 2009;5(1):19-38.
24. Wright JL, Cosio M, Churg A. Animal models of chronic obstructive pulmonary disease. Am J Physiol Lung Cell Mol Physiol. 2008;295(1): L1-L15.

25. Korsgren M, Linden M, Entwistle N, et al. Inhalation of LPS induces inflammatory airway responses mimicking characteristics of chronic obstructive pulmonary disease. Clin Physiol Funct Imaging. 2012;32(1):71-79.

26. Kharitonov SA, Sjobring U. Lipopolysaccharide challenge of humans as a model for chronic obstructive lung disease exacerbations. Contrib Microbiol. 2007;14:83-100.

27. Shapiro SD. Animal models for COPD. Chest. 2000;117(5 Suppl 1): 223S-227S

28. Alm AS, Li K, Chen H, Wang D, Andersson R, Wang X. Variation of lipopolysaccharide-induced acute lung injury in eight strains of mice. Respir Physiol Neurobiol. 2010;171(2):157-164.

29. Hakansson HF, Smailagic A, Brunmark C, Miller-Larsson A, Lal H. Altered lung function relates to inflammation in an acute LPS mouse model. Pulm Pharmacol Ther. 2012;25(5):399-406.

30. Olsson LE, Smailagic A, Onnervik PO, Hockings PD. (1)H and hyperpolarized (3)He MR imaging of mouse with LPS-induced inflammation. J Magn Reson Imaging. 2009;29(4):977-981.

31. Beckmann N, Tigani B, Sugar R, et al. Noninvasive detection of endotoxin-induced mucus hypersecretion in rat lung by MRI. Am J Physiol Lung Cell Mol Physiol. 2002;283(1):L22-L30.

32. Card JW, Zeldin DC, Bonner JC, Nestmann ER. Pulmonary applications and toxicity of engineered nanoparticles. Am J Physiol Lung Cell Mol Physiol. 2008;295(3):L400-L411.

33. Pusic K, Aguilar Z, McLoughlin J, et al. Iron oxide nanoparticles as a clinically acceptable delivery platform for a recombinant blood-stage human malaria vaccine. FASEB J. 2013 2013;27(3):1153-1166.

34. Barnes PJ. The cytokine network in chronic obstructive pulmonary disease. Am J Respir Cell Mol Biol. 2009;41(6):631-638.

35. Grenha A, Grainger CI, Dailey LA, et al. Chitosan nanoparticles are compatible with respiratory epithelial cells in vitro. Eur J Pharm Sci. 2007;31(2):73-84

36. Huang J, Zhong X, Wang L, Yang L, Mao H. Improving the magnetic resonance imaging contrast and detection methods with engineered magnetic nanoparticles. Theranostics. 2012;2(1):86-102.

37. Tong S, Hou S, Zheng Z, Zhou J, Bao G. Coating optimization of superparamagnetic iron oxide nanoparticles for high T2 relaxivity. Nano Lett. 2010;10(11):4607-4613.

38. Wang YX. Superparamagnetic iron oxide based MRI contrast agents: current status of clinical application. Quant Imaging Med Surg. 2011;1(1):35-40.

39. Rohrer M, Bauer H, Mintorovitch J, Requardt M, Weinmann HJ. Comparison of magnetic properties of MRI contrast media solutions at different magnetic field strengths. Invest Radiol. 2005;40(11):715-724.

40. Beckmann N, Tigani B, Mazzoni L, Fozard JR. MRI of lung parenchyma in rats and mice using a gradient-echo sequence. NMR Biomed. 2001;14(5):297-306.

41. Togao O, Tsuji R, Ohno Y, Dimitrov I, Takahashi M. Ultrashort echo time (UTE) MRI of the lung: assessment of tissue density in the lung parenchyma. Magn Reson Med. Nov 2010;64(5):1491-1498.

42. Conti G, Tambalo S, Villetti G, et al. Evaluation of lung inflammation induced by intratracheal administration of LPS in mice: comparison between MRI and histology. MAGMA. 2010;23(2):93-101.
International Journal of Nanomedicine

\section{Publish your work in this journal}

The International Journal of Nanomedicine is an international, peerreviewed journal focusing on the application of nanotechnology in diagnostics, therapeutics, and drug delivery systems throughou the biomedical field. This journal is indexed on PubMed Central,

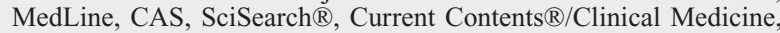

\section{Dovepress}

Journal Citation Reports/Science Edition, EMBase, Scopus and the Elsevier Bibliographic databases. The manuscript management system is completely online and includes a very quick and fair peer-review system, which is all easy to use. Visit http://www.dovepress.com/ testimonials.php to read real quotes from published authors. 